\title{
ARTICLE Leflunomide increased the renal exposure of acyclovir by inhibiting OAT1/3 and MRP2
}

\author{
Xiao-ying Liao ${ }^{1}$, Qiang-qiang Deng ${ }^{2}$, Li Han ${ }^{2,3}$, Zhi-tao Wu ${ }^{2}$, Zhao-liang Peng ${ }^{2}$, Yuan Xie ${ }^{1}$, Guang-ji Wang ${ }^{1}$, Ji-ye Aa ${ }^{1}$ and Guo-yu Pan ${ }^{2,3}$
}

\begin{abstract}
Rheumatoid arthritis patients can be prescribed a combination of immunosuppressive drug leflunomide (LEF) and the antiviral drug acyclovir to reduce the high risk of infection. Acyclovir is a substrate of organic anion transporter (OAT) $1 / 3$ and multidrug resistance-associated protein (MRP) 2. Considering the extraordinarily long half-life of LEF's active metabolite teriflunomide (TER) and the kidney injury risk of acyclovir, it is necessary to elucidate the potential impact of LEF on the disposition of acyclovir. Here we used a specific MRP inhibitor MK571 and probenecid (OAT1/3 and MRP2 inhibitor) to assess the effects of MRP2 and OAT1/3 on the pharmacokinetics and tissue distribution of acyclovir in rats. We showed that LEF and probenecid, but not MK571 significantly increased the plasma concentration of acyclovir. However, kidney and liver exposures of acyclovir were increased when coadministered with LEF, probenecid or MK571. The kidney/plasma ratio of acyclovir was increased to approximately 2-fold by LEF or probenecid, whereas it was increased to as much as 14.5-fold by MK571. Consistently, these drugs markedly decreased the urinary excretion of acyclovir. TER $(0.5-100 \mu \mathrm{mol} / \mathrm{L})$ dose-dependently increased the accumulation of acyclovir in MRP2-MDCK cells with an $\mathrm{IC}_{50}$ value of $4.91 \mu \mathrm{mol} / \mathrm{L}$. TER $(5 \mu \mathrm{mol} / \mathrm{L})$ significantly inhibited the uptake of acyclovir in hOAT1/3-HEK293 cells. These results suggest that LEF/TER increased the kidney accumulation of acyclovir by inhibiting the efflux transporter MRP2, which increased its kidney/plasma ratio and renal injury risk. However, the inhibitory effects of LEF/TER on OAT1/3 reduced the tubular cells' uptake of acyclovir and increased the plasma concentration.
\end{abstract}

Keywords: leflunomide; teriflunomide; acyclovir; organic anion transporter; multidrug resistance associated protein (MRP) 2; drugdrug interaction; pharmacokinetics

Acta Pharmacologica Sinica (2020) 41:129-137; https://doi.org/10.1038/s41401-019-0283-z

\section{INTRODUCTION}

Leflunomide (LEF) is an immunosuppressive drug that has been widely used in the clinic for the treatment of rheumatoid arthritis (RA) [1], as well as kidney/lung transplantation [2]. Most LEF is rapidly metabolized to teriflunomide (TER) in the liver and in the blood (Fig. 1) [2, 3]. The plasma concentration of TER is highly variable among patients, from 3 to $150 \mathrm{mg} / \mathrm{L}$, and the clearance of TER in the plasma is very slow, with a half-life of approximately two weeks [2]. Kidney is also responsible for its elimination [2]. We recently reported that LEF could downregulate the expression of multidrug resistance-associated protein (MRP) 2 in the liver [4]. However, its effect on the function of MRP2 still needs to be explored. In addition, TER was reported to increase the AUC of an OAT3 substrate, cefaclor [5] (https://www.accessdata.fda.gov/ drugsatfda_docs/label/2016/202992s002lbl.pdf). TER was suggested to be an inhibitor of OAT3. Because the structures of OAT1 and OAT3 are highly homologous [6], TER may also have an inhibitory effect on OAT1.

Acyclovir is a synthetic nucleoside analog for the first-line treatment of herpetic virus [7-9]. This drug is eliminated primarily in urine $(60 \%-90 \%)$ as the unchanged form [10]. Given that its renal clearance greatly exceeds the glomerular filtration rate (three-fold) $[10,11]$, tubular secretion plays an important role in its renal clearance. Acyclovir was reported to be transported by multiple transporters [12], including OAT1/3 [13, 14] and MRP2 [15]. However, previous studies have focused on the role of the uptake transporters OATs in transporting acyclovir $[14,16]$; there is limited information about efflux transporters, such as MRP2, in its renal excretion and renal accumulation. Compared with other patients, RA patients are often at a higher risk of developing infections $[17,18]$, such as herpes zoster and herpes simplex virus $[17,19]$. LEF-treated patients may be prescribed acyclovir for the treatment of herpetic infection.

Acyclovir is a common cause of kidney injury [20,21]. Crystal precipitation is widely believed to be the cause of nephrotoxicity [22]. In addition, nephrotoxicity may occur in the absence of crystal formation [23]. In other words, acyclovir could also cause direct injury to tubular cells [7, 24-26]. Therefore, reduced function of the efflux transporters may result in increased intracellular accumulation of acyclovir and subsequent detrimental nephrotoxic consequences. Because of the potential interactions of LEF with both uptake/efflux transporters, OAT1/3 and MRP2, the effect of LEF on the renal disposition of acyclovir may be complicated and deserves further investigation.

The objectives of the current work were to explore whether LEF could influence the renal accumulation of acyclovir through

\footnotetext{
${ }^{1}$ State Key Laboratory of Natural Medicines, Key Laboratory of Drug Metabolism and Pharmacokinetics, China Pharmaceutical University, Nanjing 210009 , China; ${ }^{2}$ Shanghai Institute of Materia Medica, Chinese Academy of Sciences, Shanghai 201203, China and ${ }^{3}$ University of Chinese Academy of Sciences, Beijing 100049, China Correspondence: Ji-ye Aa (jiyea@cpu.edu.cn) or Guo-yu Pan (gypan@simm.ac.cn)
} 
130

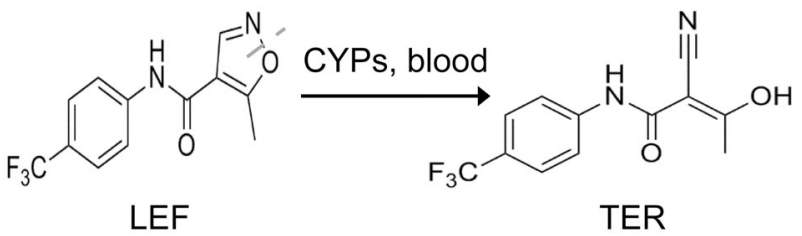

Fig. 1 The metabolism of leflunomide (LEF) to teriflunomide (TER)

coinhibiting OAT1/3 and MRP2. In this study, the specific MRP inhibitor MK571 and probenecid (inhibitor of both OAT1/3 and MRP2) were used to assess the effects of MRP2 and OAT1/3 on the pharmacokinetics and tissue distribution of acyclovir.

\section{MATERIALS AND METHODS}

Chemicals and reagents

Leflunomide (LEF, > 98.5\%), teriflunomide (TER, > 99\%), probenecid, acyclovir and G418 were obtained from Meilun Biology Technology (Dalian, China), and 6-carboxyfluorescein (6-CF) was obtained from Sigma-Aldrich (St. Louis, MO, USA). MK571, the sodium salt (98\%), was purchased from CSNpharm (Shanghai, China). TRIzol reagent, $0.05 \%$ trypsin-EDTA, DMEM high glucose, and Hanks' balanced salt solution (HBSS) were purchased from Invitrogen (Carlsbad, CA, USA). The BCA protein assay kit was from Pierce Chemical (Rockford, IL, USA). All other reagents and solvents were of analytical grade and commercially available.

\section{Animal experiments}

Male Sprague-Dawley (SD) rats weighing 200-300 g were purchased from the Animal Center of Shanghai Institute of Materia Medica (Shanghai, China). All animals were kept in the SPF class experimental animal room under conditions with a constant temperature and humidity on a $12 \mathrm{~h}$ light/dark cycle. All animal experiments were performed in accordance with the Guidance for Ethical Treatment of Laboratory Animals. The protocols involving animal experiments were reviewed and approved by the Institute of Animal Care and Use Committee (IACUC) of the Shanghai Institute of Materia Medica (Permit number: 2018-10-PGY-26).

The rats were randomly divided into four groups: (1) control group: acyclovir alone (30 mg/kg); (2) LEF group: acyclovir (30 mg/ $\mathrm{kg})+$ LEF (30 mg/kg); (3) PRO group: acyclovir $(30 \mathrm{mg} / \mathrm{kg})+$ probenecid (150 mg/kg); (4) MK571 group: acyclovir (30 mg/kg) + MK571 (10 mg/kg). Acyclovir was dissolved in isotonic saline and intravenously administered. LEF, probenecid and MK571 were all intraperitoneally injected (ip) $30 \mathrm{~min}$ before acyclovir administration.

After intravenous administration of acyclovir, blood samples were collected at 5, 10,30,60,120, 240, and $480 \mathrm{~min}$ in the four groups ( $n=4$ per group). For the urinary excretion study, urine samples ( $n=4$ per group) were collected before dosing and at different time intervals $(0-2,2-4,4-8,8-12$, and $12-24 \mathrm{~h})$, and the urine volume was recorded. The plasma concentration and the urinary concentration of acyclovir were determined by liquid chromatography-mass spectrometry tandem mass spectrometry (LC-MS/MS) (LCMS-8030; Shimadzu, Kyoto, Japan). The plasma concentration of TER was determined by an LC-MS/MS method as previously established in our lab [27].

For tissue distribution, $2 \mathrm{~h}$ after acyclovir administration, rats ( $n=3$ at each time point in each group) were sacrificed via exsanguination from the abdominal aorta under anesthesia. Livers and kidneys were rapidly dissected, washed with saline, dried and weighed. Every $200 \mathrm{mg}$ of the tissue samples was homogenized with $1 \mathrm{~mL}$ of distilled water and stored at $-80^{\circ} \mathrm{C}$ until analysis.
Blood samples were collected and centrifuged to harvest plasma samples at the same time.

Cell culture

MDCKII cells stably expressing the human transporter MRP2 (MRP2-MDCK) were kindly supplied by Prof Xiao-yan Chen (Shanghai Institute of Materia Medica, Shanghai, China). The cells were routinely cultured in DMEM medium supplemented with $10 \%$ fetal bovine serum and $100 \mathrm{U} / \mathrm{mL}$ penicillin and streptomycin.

HEK293 cells overexpressing human OAT1 (NM_153276) or human OAT3 (NM_001184732) were transfected with hOAT1pcDNA3.1 (+) or hOAT3-pcDNA3.1 (+). Control HEK293 cells were obtained in parallel via transduction of an empty pcDNA3.1(+) vector. Cell lines stably expressing empty vector (mock-HEK293) and transporters (hOAT1-HEK293 and hOAT3-HEK293) were obtained by G418 selection and then maintained in DMEM supplemented with $10 \%$ FBS and $0.5 \mathrm{mg} / \mathrm{mL} \mathrm{G} 418$ at $37^{\circ} \mathrm{C}$ with $5 \% \mathrm{CO}_{2}$.

Effects of LEF/TER on the accumulation of acyclovir in MRP2-MDCK cells

To further investigate the role of MRP2, accumulation experiments in MRP2-MDCK cells were performed as previously described [28-31]. Briefly, cells were seeded at a density of $2 \times 10^{5}$ cells/well on a 24-well plate. After $48 \mathrm{~h}$ of seeding, the cells were washed twice with warm $\left(37^{\circ} \mathrm{C}\right) \mathrm{HBSS}$ and then preincubated with $0.3 \mathrm{~mL}$ of HBSS solution for $15 \mathrm{~min}$ at $37^{\circ} \mathrm{C}$. Then, acyclovir $(200 \mu \mathrm{mol} / \mathrm{L})$ in the presence or absence of test compounds was incubated for $60 \mathrm{~min}$. At the end of the studies, the medium was aspirated, and the cells were washed three times with ice-cold HBSS. Subsequently, the samples were frozen at $-80^{\circ} \mathrm{C}$ before LC-MS/MS analysis. The $\mathrm{IC}_{50}$ value for LEF/TER on the accumulation of acyclovir in MRP2-MDCK cells was determined from the maximum and minimum extremes of the relevant non-linear regression plot by GraphPad Prism 5 (San Diego, CA, USA) (maximal inhibition = $100 \%$, non-inhibited control $=0 \%$ ).

\section{Transcellular transport assays}

MRP2-MDCK cells at a density of $3 \times 10^{5}$ cells $/ \mathrm{cm}^{2}$ were grown on a polycarbonate membrane filter membrane on Transwell inserts (0.4 $\mu \mathrm{m}$ pore size, $6.25 \mathrm{~mm}$ diameter; Costar, Corning, NY, USA) for $5 \mathrm{~d}$, and monolayers with a TEER-value above $420 \Omega / \mathrm{cm}^{2}$ were utilized for the studies. Before starting the transport studies, the apical (A) and basolateral (B) chambers were washed twice with prewarmed $\mathrm{HBSS}\left(37^{\circ} \mathrm{C}\right)$, and then, the cells were equilibrated for $30 \mathrm{~min}$ in the presence or absence of inhibitors. HBSS containing acyclovir $(10 \mu \mathrm{mol} / \mathrm{L})$ with or without inhibitors was added to the donor side (either the apical or basolateral side). Aliquots $(50 \mu \mathrm{L})$ were collected from the acceptor compartment at $60 \mathrm{~min}$ for LCMS/MS analysis.

Inhibitory effect of LEF/TER on OAT1/3 in hOAT1-HEK293 and hOAT3-HEK293 cells

For inhibition studies, 6-CF was used as the specific fluorescent substrate of OAT1 and OAT3 [32]. The 6-CF $(5 \mu \mathrm{mol} / \mathrm{L})$ uptake process with or without LEF $(1-100 \mu \mathrm{mol} / \mathrm{L})$ or TER $(0.5-50 \mu \mathrm{mol} / \mathrm{L})$ was conducted as described above, with the exception that HBSS containing probenecid $(200 \mu \mathrm{mol} / \mathrm{L})$ was used as a positive inhibitor, and the incubation time was set at $10 \mathrm{~min}$. At the end of the uptake studies, the medium was aspirated, and the cells were washed three times with ice-cold HBSS. Then, the cells were lysed with $300 \mu \mathrm{L}$ of $0.1 \mathrm{~mol} / \mathrm{L}$ sodium hydroxide. The content of 6CF was measured with excitation and emission wavelengths at 485 and $528 \mathrm{~nm}$ with a Microplate Reader (Bio-Tek Instruments, USA), respectively. The protein content of the solubilized cells was determined using a BCA protein assay kit. The fluorescence 
intensity was normalized to total protein. $I C_{50}$ values were calculated using nonlinear regression with an appropriate model by GraphPad Prism 5.

The acyclovir uptake process was conducted as described above, with the exception that HBSS containing LEF $(10 \mu \mathrm{mol} / \mathrm{L})$, TER $(5 \mu \mathrm{mol} / \mathrm{L})$ and probenecid $(200 \mu \mathrm{mol} / \mathrm{L})$ was used as inhibitors, and the incubation time was set at $30 \mathrm{~min}$. The uptake medium containing $200 \mu \mathrm{mol} / \mathrm{L}$ acyclovir, with or without the inhibitors above, was then added to each well. At the end of the study, the acyclovir content was determined by LCMS/MS.

LEF/TER cellular uptake assays

To estimate whether TER was a substrate of OAT1 or OAT3, cellular accumulation for TER was performed in hOAT1-HEK293 and hOAT3-HEK293 cells. The uptake process was conducted as described above, with the exception that HBSS containing TER $(20 \mu \mathrm{mol} / \mathrm{L})$ was used as the incubation media and the incubation time was set for $20 \mathrm{~min}$. The uptake medium containing TER $(20 \mu \mathrm{mol} / \mathrm{L})$ with or without probenecid $(200 \mu \mathrm{mol} / \mathrm{L})$ was then added to each well. The uptake procedure was also terminated as described above. The TER content was analyzed by LC-MS/MS.

\section{Sample preparation}

For acyclovir, urine samples were diluted 50 times with water. A $50 \mu \mathrm{L}$ aliquot of a sample (plasma, diluted urine, tissue homogenate samples or cell lysates) was vortexed with $200 \mu \mathrm{L}$ of methanol containing internal standard (fluconazole) for $3 \mathrm{~min}$ and then centrifuged at $12000 \times g$ for $20 \mathrm{~min}$. For plasma and urine samples, the upper layer was transferred into another polythene tube and diluted 4-fold with ultrapure water. Finally, a $5 \mu \mathrm{L}$ aliquot was used for the LC-MS/MS analysis.

\section{LC-MS/MS analysis}

Acyclovir was analyzed with electrospray ionization (ESI) on a Shimadzu LCMS-8030 triple quadrupole system (Shimadzu Corp, Kyoto, Japan) and separated using a Welch Ultimate AQ-C18 column $(150 \mathrm{~mm} \times 4.6 \mathrm{~mm}, 5 \mu \mathrm{m})$. Multiple reaction monitoring (MRM) was used to quantify compounds in the positive ion mode ( $\mathrm{m} / \mathrm{z}$ 226.2-152.1 for acyclovir and $\mathrm{m} / \mathrm{z}$ 307.1-220.1 for the internal standard fluconazole). The mobile phase was methanol (A) and $0.1 \%$ formic acid in water (B) at a flow rate of $0.4 \mathrm{~mL} / \mathrm{min}$ with the gradient conditions as follows: $0-1 \mathrm{~min}, 40 \% \mathrm{~B} ; 1-2 \mathrm{~min}, 40 \%-10 \%$ $\mathrm{B}$; 2-3 $\mathrm{min}, 10 \% \mathrm{~B} ; 3-3.1 \mathrm{~min}$, return to $40 \% \mathrm{~B}$; and $3.1-7 \mathrm{~min}$ reequilibrium.

Data analysis

The permeability coefficients of acyclovir $\left(P_{\text {app }}\right)$ and the efflux ratio (ER) were calculated according to the following equation:

$P_{\text {app }}(\mathrm{cm} / \mathrm{s})=\left(C_{\mathrm{t}} \times V\right) /\left(\right.$ Area $\left.\times C_{0} \times t\right)$

$P_{\text {app }}(\mathrm{cm} / \mathrm{s})=\left(C_{\mathrm{t}} \times V\right) /\left(\right.$ Area $\left.\times C_{0} \times t\right)$, where $C_{\mathrm{t}}=$ the concentration of acyclovir on the receiver side, $V=$ the loading volume on the receiver side, Area $=$ membrane surface area $\left(0.33 \mathrm{~cm}^{2}\right), C_{0}=$ the initial concentration on the donor side, and $t=$ incubation time.

$$
\mathrm{ER}=P_{\mathrm{B} \text { to } \mathrm{A}} / P_{\mathrm{A} \text { to } \mathrm{B}}
$$

where $P_{\mathrm{A} \text { to } \mathrm{B}}$ and $P_{\mathrm{B}}$ to A represent the permeability from the apical to basolateral sides and from the basolateral to apical sides, respectively.

PK parameters were calculated in a non-compartmental analysis utilizing WinNonlin software (Pharsight 6.2, NC, USA). The plasma clearance $\left(C_{P}\right)$ of acyclovir was calculated using the following equation:

$$
\mathrm{CL}_{\mathrm{P}}=\text { Dose } / \mathrm{AUC}_{0-\infty}
$$

The $\mathrm{AUC}_{0-\infty}$ is the area under the plasma concentration-time profile after iv administration.

The renal clearance $\left(C_{R}\right)$ of acyclovir was calculated using the following equation:

$\mathrm{CL}_{\mathrm{R}}=A_{\text {total }} / \mathrm{AUC}_{0-\infty}$

$A_{\text {total }}$ is the total cumulative amount of acyclovir excreted in urine over $24 \mathrm{~h}$.

The data are presented as the mean \pm SD. Student's two-tailed unpaired $t$-test was used for comparisons between two groups using GraphPad Prism 5. $P<0.05$ was considered to be statistically significant.

\section{RESULTS}

LEF increased plasma exposure of acyclovir and decreased its urinary excretion

Probenecid was used as a conventional inhibitor of OAT1/3 and MRP2. The specific MRP inhibitor MK571 was employed to investigate its pure inhibitory effect on MRP2.

Coadministration of LEF increased the $\mathrm{AUC}_{0-\infty}$ of acyclovir by $53 \%(P<0.001)$, decreased its total body clearance by $45 \%(P<$ $0.05)$, and prolonged the mean residence time (MRT) by $33 \%$ $(P<0.05)$ compared to single acyclovir administration (Fig. 2a, Table 1). Probenecid reduced the total body clearance of acyclovir by $72 \%$ and significantly increased its $A U C_{0-\infty}$ by $2.52-$ fold $(P<0.001)$ (Table 1$)$. The MRP2 inhibitor MK571 had no effect on the plasma concentration and plasma clearance of acyclovir.

The plasma concentration of TER was detected in the LEF group. During $0-8 \mathrm{~h}$ after acyclovir administration, the mean plasma concentration of TER varied from 25.9 to $37.8 \mu \mathrm{g} / \mathrm{mL}$ (Fig. 2b). The peak concentration reached at $2 \mathrm{~h}$ and did not change obviously until acyclovir was almost completely eliminated $(8 \mathrm{~h}$ after administration).

For administration of acyclovir alone, $78.4 \%$ of the dose was recovered from the rat urine through 24 -h cumulative urinary excretion of acyclovir (Fig. 3). The combination with LEF reduced the cumulative urinary excretion of acyclovir to $42.2 \%(P<0.001)$ (Fig. 3), and $\mathrm{CL}_{R}$ was reduced to $34.6 \%(P<0.001)$ (Table 1). MK571 and probenecid showed stronger inhibition of urinary excretion and reduced $\mathrm{CL}_{\mathrm{R}}$ to $32.9 \%(P<0.001)$ and $9.8 \% \quad(P<0.001)$ (Table 1).

LEF increased the concentration and tissue/plasma ratio of acyclovir in liver and kidney

The liver and kidney concentrations of acyclovir in the coadministration groups were significantly increased compared to acyclovir administration alone at $2 \mathrm{~h}$ (Fig. 4). LEF increased the accumulation of acyclovir in the liver and kidney to 3.2-fold $(P<0.05)$ and 4.2 -fold $(P<0.05)$, respectively. Coadministration of MK571 or probenecid had a stronger effect on the accumulation of acyclovir in the liver and kidney at $2 \mathrm{~h}$, but the increased acyclovir concentration in kidney was proportional to its increased plasma concentrations in the LEF and PRO groups (Fig. 4a, b). The tissue/ plasma concentration ratios in the liver and kidney after LEF treatment were increased to 1.71 -fold $(P<0.05)$ and 2.23-fold $(P<$ $0.05)$, respectively. In the PRO group, the values were 1.51 -fold and 2.45-fold, respectively. In contrast, MK571 only significantly increased acyclovir concentrations in the kidney and liver but did not significantly change the plasma exposure. Therefore, probenecid's effect on the tissue/plasma concentration ratio in the 
a

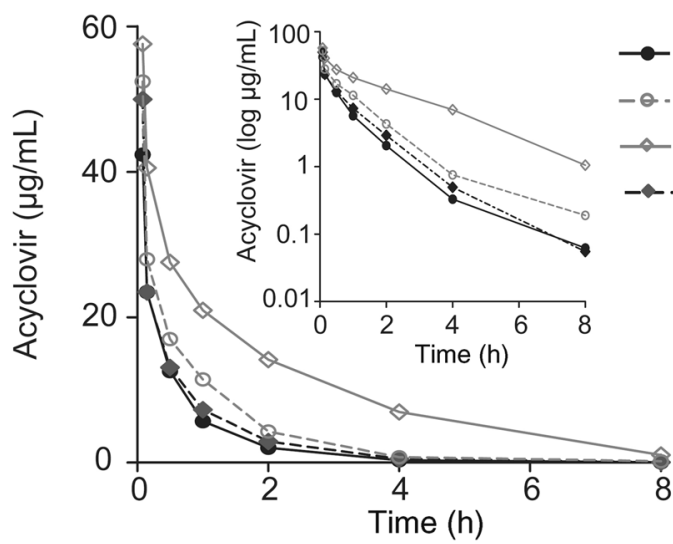

b

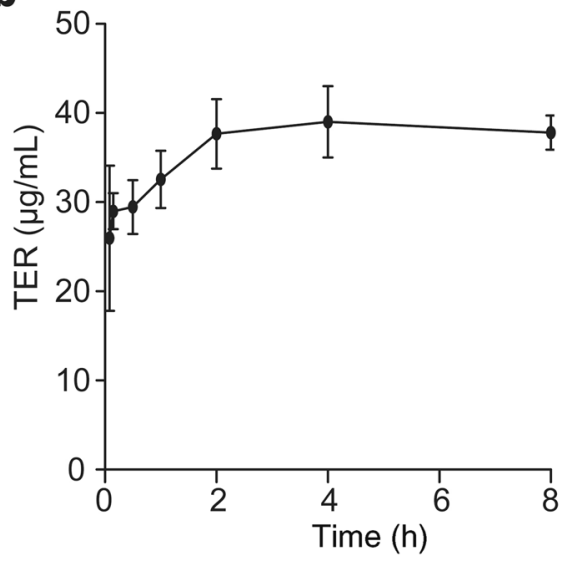

Fig. 2 Mean plasma concentration-time curve of acyclovir (a) and TER (b) (LEF group) after acyclovir administration. Insets: mean log plasma concentration-time curves of acyclovir in different groups (a). Data are expressed as the mean in a to clearly show the data, and data are shown as the mean \pm SD in b. $(n=4)$

Table 1. Pharmacokinetic parameters of acyclovir after intravenous administration

\begin{tabular}{lcccc}
\hline Group & Acyclovir alone & Combined with LEF & Combined with probenecid & Combined with MK571 \\
\hline AUC $_{0-\infty}\left(\mu \mathrm{g} / \mathrm{mL}^{*} \mathrm{~h}\right)$ & $25.8 \pm 3.02$ & $39.7 \pm 2.78^{* * *}$ & $91.4 \pm 11.7^{* * *}$ & $30.6 \pm 4.53$ \\
$T_{1 / 2}(\mathrm{~h})$ & $1.06 \pm 0.08$ & $1.16 \pm 0.06$ & $1.58 \pm 0.38^{*}$ & $1.03 \pm 0.17$ \\
$\mathrm{MRT}(\mathrm{h})$ & $0.77 \pm 0.1$ & $1.01 \pm 0.1^{*}$ & $2.1 \pm 0.46^{* * *}$ & $0.83 \pm 0.14$ \\
$\mathrm{CL}(\mathrm{mL} / \mathrm{h} / \mathrm{kg})$ & $1173 \pm 141$ & $759 \pm 55^{* *}$ & $332 \pm 45^{* * *}$ & $995 \pm 133$ \\
$\mathrm{CL}_{\mathrm{R}}(\mathrm{mL} / \mathrm{h} / \mathrm{kg})$ & $925.8 \pm 174$ & $320.5 \pm 42.9^{* * *}$ & $90.7 \pm 30.1^{* * *}$ & $305.5 \pm 91.9^{* * *}$ \\
\hline
\end{tabular}

Note: Values represent the mean \pm SD $(n=4)$. Statistics were conducted using a two-tailed unpaired $t$-test. ${ }^{*} P<0.05,{ }^{* *} P<0.01,{ }^{* * *} P<0.001$ vs. single administration

$T_{1 / 2}$ half-life, $M R T$ mean residence time, $A \cup C_{0-\infty}$ area under the plasma concentration-time curve from time zero extrapolated to infinity, $C L_{R}$ renal clearance, $C L_{P}$ plasma clearance

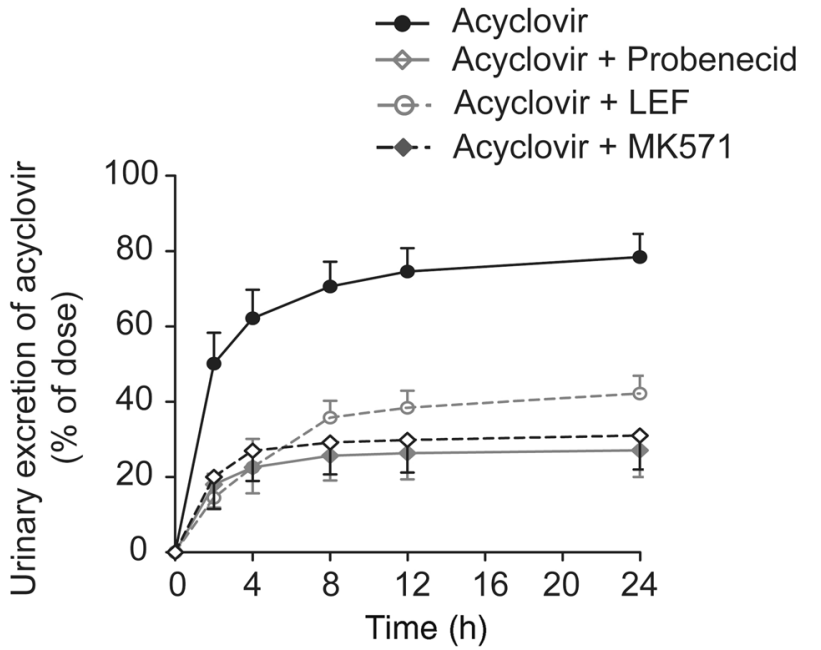

Fig. 3 The cumulative urinary excretion of acyclovir after administration of acyclovir with or without LEF, probenecid or MK571. Data are expressed as the mean \pm SD. $(n=4)$

liver and kidney was similar to LEF, and MK571 treatment increased the ratios to 5.8 -fold $(P<0.001)$ and 14.5 -fold $(P<$ 0.01 ), respectively (Fig. $4 c, d$ ).
TER inhibited acyclovir transcellular transport and increased intracellular accumulation in MRP2-MDCK cells

To understand the mechanism of the interaction between LEF and acyclovir, we examined the effect of LEF/TER on the cellular accumulation of acyclovir in MRP2-MDCK cells. A typical MRP2 inhibitor, MK571 $(20 \mu \mathrm{mol} / \mathrm{L})$, increased acyclovir accumulation in MRP2-MDCK by $3-4$-fold $(P<0.001)$ (Fig. $5 a)$. TER, but not LEF, increased the accumulation of acyclovir in MRP2-MDCK cells in a concentration-dependent manner, with an $\mathrm{IC}_{50}$ of $4.91 \mu \mathrm{mol} / \mathrm{L}$ (Fig. 5b). Consistent with the accumulation assay results, acyclovir in MRP2-MDCK cells showed greater permeability in the $B$ to $A$ direction compared with that in the $A$ to $B$ direction (Fig. $5 \mathrm{C}$ ). The ER value of acyclovir was 2.27. TER $(10 \mu \mathrm{mol} / \mathrm{L})$ and MK571 reduced the permeability of acyclovir (the ER value was reduced to 1.46$)$.

Inhibitory activity of LEF/TER in hOAT1-HEK293 and hOAT3HEK293 cells

We investigated whether LEF/TER had a potential inhibitory effect on OAT1/3. TER, the main LEF metabolite, inhibited the uptake of 6-CF, a fluorescent substrate of OAT1/3, in hOAT1-/3-HEK293 cells with $\mathrm{IC}_{50}$ values of $3.39 \mu \mathrm{mol} / \mathrm{L}$ and $0.87 \mu \mathrm{mol} / \mathrm{L}$ (Fig. $6 \mathrm{c}$, d). The prodrug LEF showed a weaker inhibition on OATs. The estimated $\mathrm{IC}_{50}$ value of $\mathrm{LEF}$ on OAT3 was $4.1 \mu \mathrm{mol} / \mathrm{L}$, but there was no significant inhibitory effect on OAT1 (Fig. 6a, b).

Furthermore, to investigate whether TER was a substrate of OAT1/3, TER uptake analysis was conducted on OAT1/3-trans- 
a

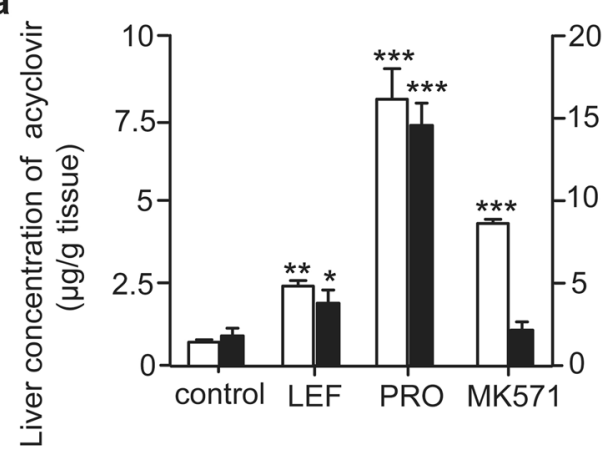

C

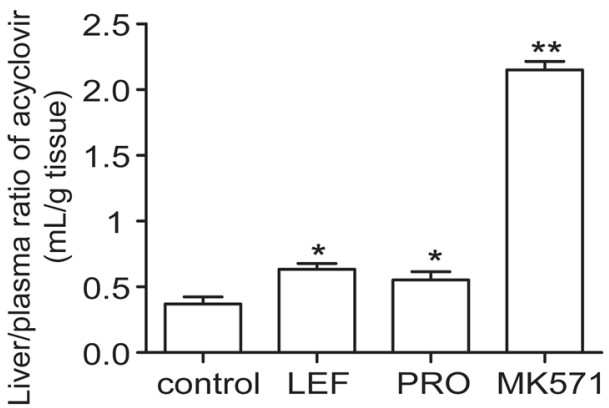

b

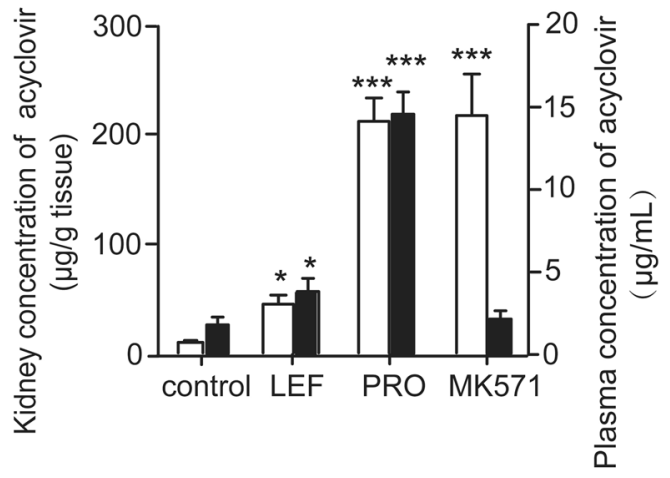

d

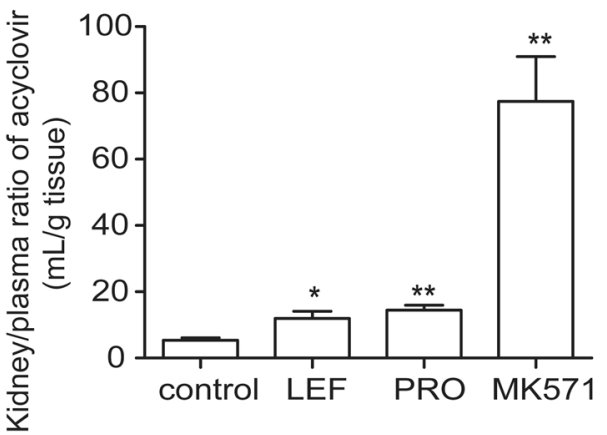

Fig. 4 Effects of LEF, probenecid (PRO) and MK571 on the tissue distribution and the tissue/plasma concentration ratios of acyclovir. a and b Open columns on the left represent the acyclovir concentrations in liver and kidney, respectively. The black columns on the right are the plasma acyclovir concentrations. c liver/plasma concentration ratio, (d) kidney/plasma concentration ratio. Each bar represents the mean \pm SD in triplicate. $\left(n=3,{ }^{*} P<0.05,{ }^{* *} P<0.01,{ }^{* *} P<0.001\right.$ compared to the control)

fected cells. The transfected cells could not increase the cellular uptake of TER compared to mock cells, and the presence of probenecid did not alter the intracellular uptake of TER in both transfected cell lines (Fig. 7a), indicating that TER was not a substrate of OAT1/3. Furthermore, acyclovir uptake assays were performed using these transfected cells (Fig. 7b). The uptake of acyclovir was higher in hOAT1-HEK293 and hOAT3-HEK293 cells than mock-HEK293 cells. Similar to the OAT inhibitor probenecid, TER $(5 \mu \mathrm{mol} / \mathrm{L})$ inhibited OAT-mediated acyclovir transport, while LEF $(10 \mu \mathrm{mol} / \mathrm{L})$ only inhibited OAT3-mediated acyclovir transport.

\section{DISCUSSION}

OAT1/3 on the renal basolateral membrane are responsible for the uptake of anionic drugs from the blood, while apical transporters, such as MRP2, ensure their efflux into the tubular lumen [33-35]. Inhibition of uptake or efflux transporters by a coadministered drug can alter their renal distribution. Previous studies have focused on the basolateral uptake of acyclovir by OAT1/3 in vitro and in vivo $[13,14]$. No in vivo studies have been conducted on the contribution of MRP2 to acyclovir disposition.

In the present study, the inhibitory effect of LEF and its metabolite TER on OAT1/3 was evaluated. Because LEF will be converted to its active metabolite TER in a very short time in vivo $[2,27]$, TER was employed to assess the impact of LEF on the related transporters in subsequent in vitro studies. The calculated $\mathrm{IC}_{50}$ values of TER on OAT1 and OAT3 were $3.39 \mu \mathrm{mol} / \mathrm{L}$ and 0.87 $\mu \mathrm{mol} / \mathrm{L}$, respectively (Fig. $6 \mathrm{c}$, d). The inhibition of TER on OAT3 was stronger than that on OAT1 (Fig. 6). At the same time, TER inhibited the efflux of acyclovir in MRP2-MDCK cells in a concentration-dependent manner with an $\mathrm{IC}_{50}$ value of 4.91 $\mu \mathrm{mol} / \mathrm{L}$ (Fig. 5). To the best of our knowledge, this is the first study to identify TER, not LEF, as a potent MRP2 inhibitor.
In general, inhibition of renal uptake transporters is believed to reduce the potential nephrotoxicity of their substrates by reducing renal accumulation. For example, probenecid could reduce the nephrotoxicity of aristolochic acid I (AAI) and cidofovir in the clinic by inhibiting OAT1/3 [36, 37]. However, the success of probenecid with AAI and cidofovir came from the fact that neither AAI nor cidofovir is a MRP2 substrate [31, 38]. In fact, for drugs that are both substrates of efflux/influx transporters, caution should be taken in interpreting the results.

If LEF/TER only inhibited the uptake transporters OAT1/3, the acyclovir concentration in the kidney should be reduced. However, the kidney concentration of acyclovir (at $2 \mathrm{~h}$ ) and the kidney/plasma ratio (at $2 \mathrm{~h}$ ) were significantly increased by coadministration of LEF. A similar effect was observed with probenecid, an inhibitor of OAT1/3 and MRP2 (Fig. 4). The results implied that the efflux transporters for transporting acyclovir may be inhibited by LEF or probenecid, as well as uptake transporters. The TER kidney concentration at that time was determined to be $70 \mu \mathrm{mol} / \mathrm{g}$ tissue (data not shown), which was much higher than its OAT1/3 and MRP2 IC 50 values. In addition, LEF increased the liver concentration (at $2 \mathrm{~h}$ ) and liver/plasma concentration ratio (at $2 \mathrm{~h}$ ) of acyclovir, which might also result from the inhibition of MRP2, located in the canalicular membrane of hepatocytes.

To clarify the contribution of MRP2 to acyclovir disposition in the kidney, the specific MRP inhibitor MK571 was employed. Notably, LEF and probenecid increased the $\mathrm{AUC}_{0-\infty}$ of acyclovir and decreased its renal clearance, while the plasma concentration was not changed by MK571 (Fig. 2 and Table 1). Unlike LEF and probenecid, MK571 increased the kidney concentration of acyclovir without any change to its plasma concentration, which led to an almost 14.5-fold increase in its kidney/plasma ratio (for LEF and probenecid, the increase in kidney/plasma ratio was approximately 2 -fold) (Fig. 4d). The reason for this phenomenon 
a

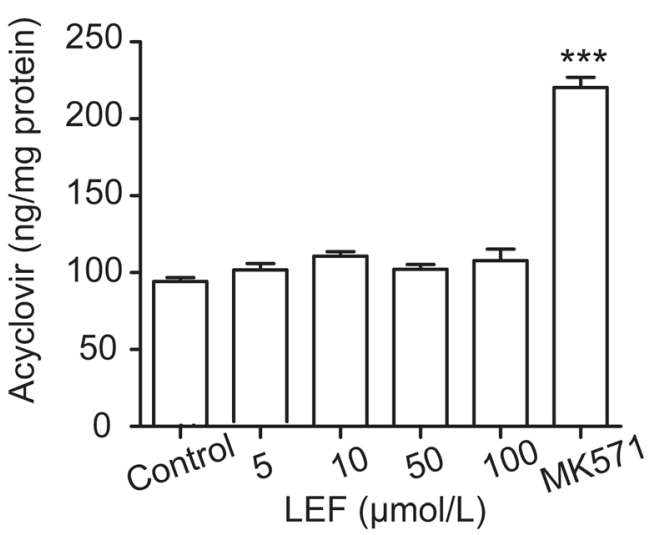

C
MRP2

MRP2

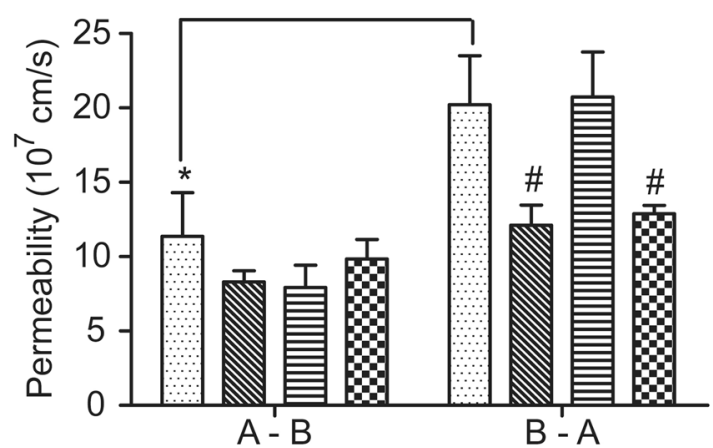

b

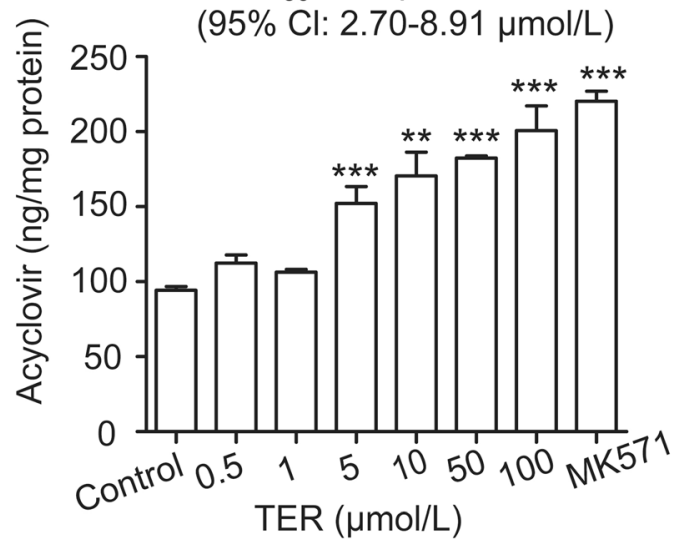

MRP2

$\mathrm{IC}_{50} 4.91 \mu \mathrm{mol} / \mathrm{L}$

$95 \% \mathrm{Cl}: 2.70-8.91 \mu \mathrm{mol} / \mathrm{L})$

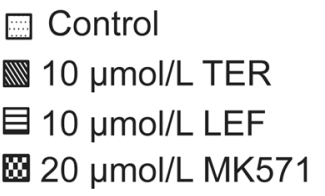

Fig. 5 Cellular accumulation and permeability of acyclovir in MRP2-MDCK cells. Effects of LEF $(5-100 \mu \mathrm{mol} / \mathrm{L})(\mathbf{a})$ and TER (0.5-100 $\mu \mathrm{mol} / \mathrm{L})$ (b) on acyclovir accumulation in the MRP2-MDCK cells, and MK571 $(20 \mu \mathrm{mol} / \mathrm{L})$ was used as the positive control. c The permeability of acyclovir $(10 \mu \mathrm{mol} / \mathrm{L})$ in the absence or presence of TER $(10 \mu \mathrm{mol} / \mathrm{L}), \mathrm{LEF}(10 \mu \mathrm{mol} / \mathrm{L})$ and MK571 $(20 \mu \mathrm{mol} / \mathrm{L})$ in the transport studies in MRP2-MDCK cells. Data are presented as the mean \pm SD of experiments performed in triplicate. Asterisk $\left(^{*}\right)$ represents the significant difference in acyclovir permeability compared A-B to B-A $(P<0.05)$. \# represents the significance in $P_{\mathrm{B}}$ to $\mathrm{A}$ of acyclovir compared to the control $(P<0.05)$

could be explained by the overall intrinsic clearance concept introduced by Sugiyama [39, 40]: For drugs that are influenced by both efflux and uptake transporters, particularly for those whose efflux clearance into bile/urine is greater than the basolateral efflux (efflux into the blood), the reduction of the uptake capacity may affect the plasma concentration-time profile more than the target tissue concentrations. In contrast, if the efflux process is blocked, the target tissue concentration will be dramatically increased, while the plasma concentration is not significantly affected [41]. In this study, MK571 increased acyclovir kidney concentration, but it did not change its plasma exposure, which was consistent with the fact that only MRP transporters were inhibited. This finding implied that the efflux transporters MRP may play a critical role in acyclovir kidney disposition, such as MRP2, which may be the critical factor in determining the effect of LEF and probenecid on acyclovir disposition in the kidney. From another perspective, it is clear that the increase of acyclovir plasma exposure in combination with LEF and probenecid came from the inhibition of OAT1/3. Meanwhile, the increased kidney/ plasma ratios of acyclovir after LEF or probenecid treatment were much lower than that by MK571, which implied that the reduced function of the uptake transporters OAT1/3 partly alleviated the renal accumulation of acyclovir via MRP2 inhibition. The elevated kidney concentration of acyclovir after LFE administration was a combined effect of OATs and MRP2 (illustrated in Fig. 8). LEF/TER is also a substrate of BCRP [42], and the inhibition of BCRP may contribute to the renal accumulation of acyclovir by LEF. However, the MK571 inhibition results suggested that MRPs dominated the cumulative urinary excretion of acyclovir (Fig. 3).

In our study, acyclovir disposition in the kidney was influenced by both efflux and uptake transporters. The potential DDI risk is relatively easy to estimate if only one type of transporter is involved. For example, MRP2 mutation is associated with impaired renal elimination and nephrotoxicity of methotrexate [43]. For drugs that are multiple-transporter perpetrators such as LEF, attention should be paid to assess the different contribution of efflux/influx transporters before making conclusions, especially for drugs with narrow therapeutic windows. For example, nonsteroidal anti-inflammatory drugs (NSAIDs), which have also been reported to be nephrotoxic [44, 45], are well-known OAT1/3 and MRP substrates [46, 47]. The combination of NSAIDs with methotrexate could result in serious toxicity, including an increased risk of nephrotoxicity [48, 49]. An increased risk of acute kidney injury was also observed with the concomitant use of NSAIDs with acyclovir or valacyclovir (a prodrug of acyclovir) [20,50].

In conclusion, our work indicated that LEF increased the systemic exposure of acyclovir and increased the tissue/plasma ratios in rat liver and kidney. TER, the major metabolite of LEF, is a potent MRP2 and OAT1/3 inhibitor. The results suggested that LEF increased the renal exposure of acyclovir mainly by inhibiting MRP2, while it inhibited the uptake transporters OAT1/3, which resulted in increasing plasma exposure of acyclovir. 


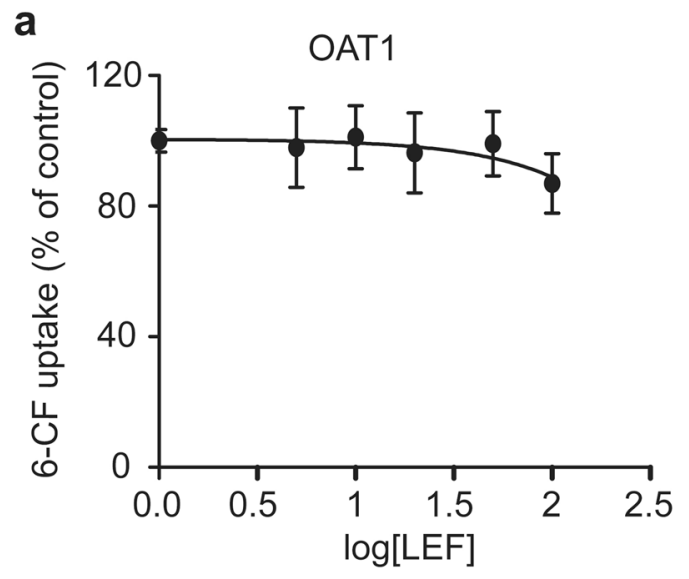

b
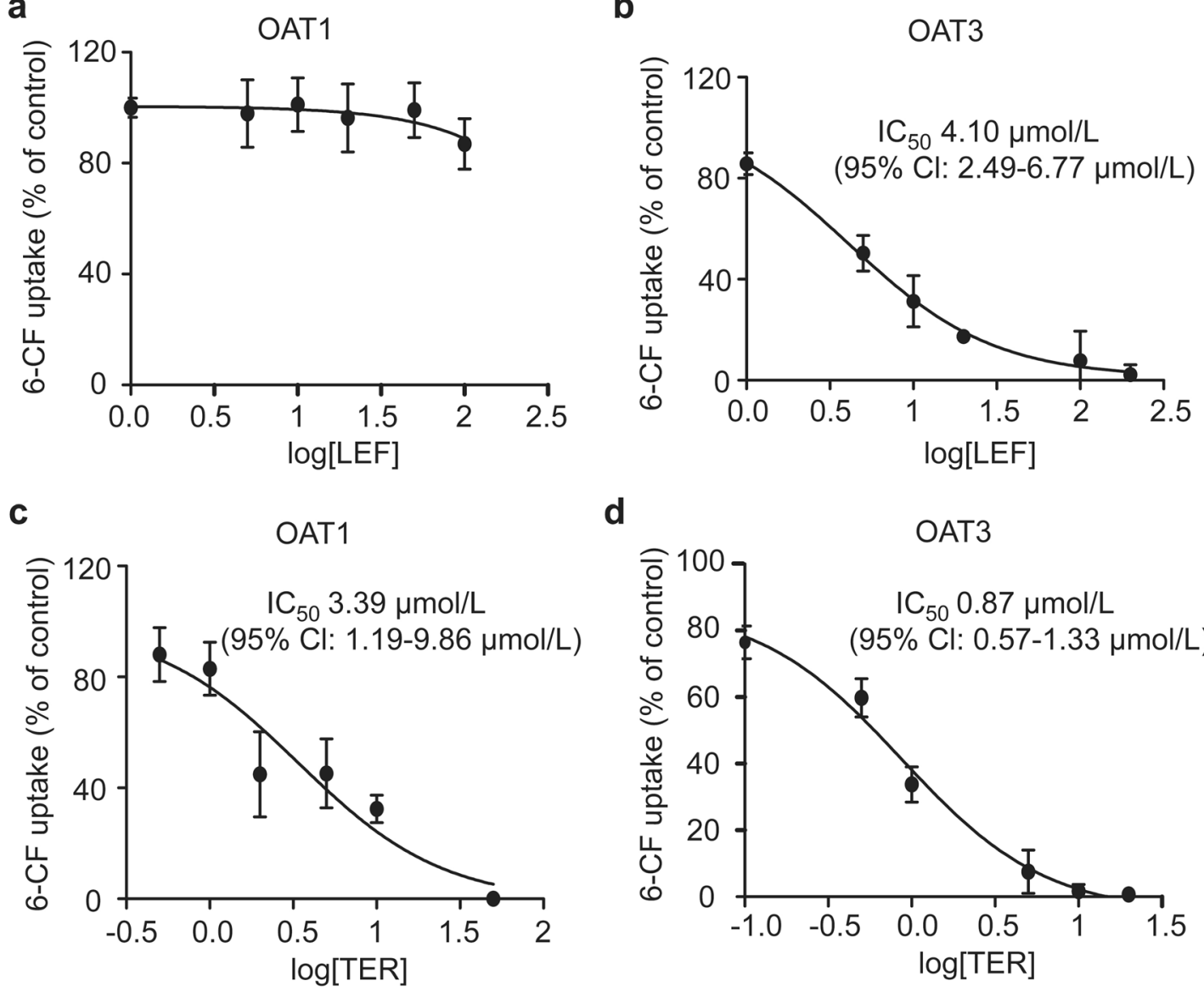

d

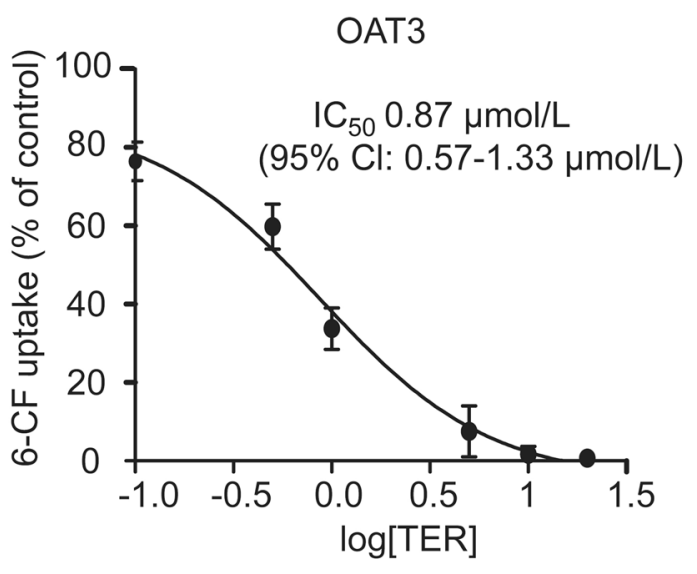

Fig. 6 Inhibitory effect of LEF $(1-100 \mu \mathrm{mol} / \mathrm{L})$ and TER $(0.5-50 \mu \mathrm{mol} / \mathrm{L})$ on $6-\mathrm{CF}$ uptake in hOAT1/3-HEK293 cells. Concentration-dependent inhibition of 6-CF uptake assays for the quantification of the inhibitory effect of LEF on OAT1 (a) and OAT3 (b) and the corresponding inhibition of TER on OAT1 (c) and OAT3 (d). Data are shown as the mean \pm SD of experiments performed in triplicate
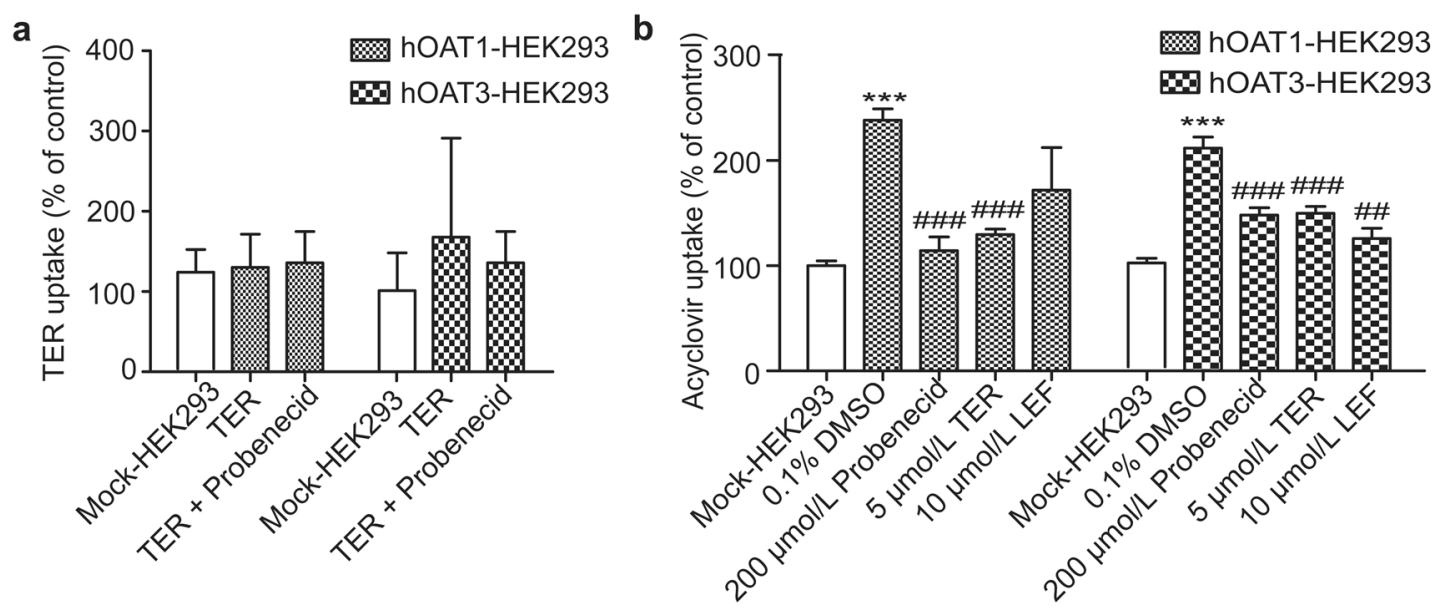

Fig. 7 Intracellular uptake of TER (a) and the effect of TER on acyclovir uptake in hOAT1/3-transfected cells (b). The values shown are the

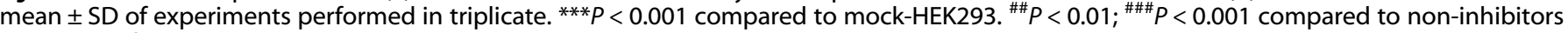
in the transfected cell lines

\section{ACKNOWLEDGEMENTS}

The authors thank Prof Xiao-yan Chen (Shanghai Institute of Materia Medica, Chinese Academy of Science, Shanghai, China) for providing the MRP2-MDCK cells. This work was supported by "Organ Reconstruction and Manufacturing" Strategic Priority Research Program of the Chinese Academy of Sciences (Grant: XDA16020205), the
National Natural Science Foundation of China (No_81872927), the National Key Special Project of Science and Technology for Innovation Drugs of China (No 2015zx09501001), and the Independent Deployment Program of the Institute of Pharmaceutical Innovation of the Chinese Academy of Sciences (Grant: CASIMM0120184005). 


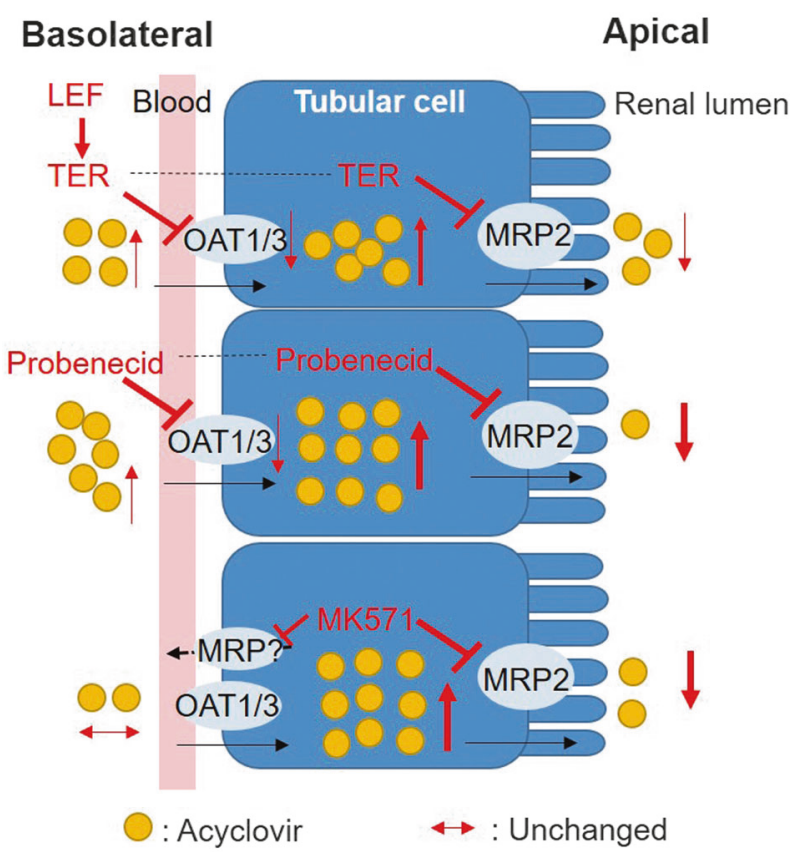

Fig. 8 Schematic diagram illustrating the inhibitory mechanisms of LEF, probenecid and MK571 on the transport of acyclovir. LEF is rapidly converted to TER in vivo, which can inhibit the uptake and efflux of acyclovir mediated by OAT1/3 and MRP2. Probenecid, at a high dose in this study, exhibited a stronger inhibitory effect on OAT1/3 and MRP2. MK571 did not have an obvious effect on OAT1/3 and inhibited the efflux of acyclovir into the urine. The inhibition of OAT $1 / 3$ reduced the uptake of acyclovir into the cells and increased the plasma concentration, while the inhibition of MRP2 increased the accumulation in the tubular cells and reduced its urinary excretion

\section{AUTHOR CONTRIBUTIONS}

GYP and JYA designed the paper. XYL, QQD, and LH carried out the experiments. ZLP, ZTW, YX, and GJW analyzed the data. XYL and GYP prepared the paper. JYA, GYP, and GJW critically revised the paper.

\section{ADDITIONAL INFORMATION}

Conflict of interest: The authors declare that they have no conflict of interest.

\section{REFERENCES}

1. Smolen JS, Landewe R, Bijlsma J, Burmester G, Chatzidionysiou K, Dougados M, et al. EULAR recommendations for the management of rheumatoid arthritis with synthetic and biological disease-modifying antirheumatic drugs: 2016 update. Ann Rheum Dis. 2017;76:960-77.

2. Rozman. Clinical pharmacokinetics of leflunomide. Clin Pharmacokinet. 2002;41: 421-30.

3. Kalgutkar AS, Nguyen HT, Vaz ADN, Doan A, Dalvie DK, McLeod DG, et al. In vitro metabolism studies on the isoxazole ring scission in the anti-inflammatory agent lefluonomide to its active alpha-cyanoenol metabolite A771726. Drug Metab Dispos. 2003;31:1240-50.

4. Wang L, Ma LL, Lin Y, Liu X, Xiao L, Zhang Y, et al. Leflunomide increases hepatic exposure to methotrexate and its metabolite by differentially regulating multidrug resistance-associated protein Mrp2/3/4 transporters via peroxisome proliferator-activated receptor alpha activation. Mol Pharmacol. 2018;93:563-74.

5. Ueo $\mathrm{H}$, Motohashi $\mathrm{H}$, Katsura $\mathrm{T}$, Inui $\mathrm{K}$. Human organic anion transporter hOAT3 is a potent transporter of cephalosporin antibiotics, in comparison with hOAT1. Biochem Pharmacol. 2005;70:1104-13.

6. Srimaroeng C, Perry JL, Pritchard JB. Physiology, structure, and regulation of the cloned organic anion transporters. Xenobiotica 2008;38:889-935.

7. Chavez-Iniguez JS, Medina-Gonzalez R, Aguilar-Parra L, Torres-Vazquez EJ, Maggiani-Aguilera P, Cervantes-Perez E, et al. Oral acyclovir induced hypokalemia and acute tubular necrosis a case report. BMC Nephrol. 2018;19:324.
8. Piret J, Boivin G. Antiviral resistance in herpes simplex virus and varicella-zoster virus infections: diagnosis and management. Curr Opin Infect Dis. 2016;29:654-62.

9. Mercier-Darty M, Boutolleau D, Lepeule R, Rodriguez C, Burrel S. Utility of ultradeep sequencing for detection of varicella-zoster virus antiviral resistance mutations. Antivir Res. 2018;151:20-3.

10. de Miranda P, Good SS, Krasny HC, Connor JD, Laskin OL, Lietman PS. Metabolic fate of radioactive acyclovir in humans. Am J Med. 1982;73:215-20.

11. Laskin OL, de Miranda P, King DH, Page DA, Longstreth JA, Rocco L, et al. Effects of probenecid on the pharmacokinetics and elimination of acyclovir in humans. Antimicrob Agents Chemother. 1982;21:804-7.

12. De Bony F, Tod M, Bidault R, On NT, Posner J, Rolan P. Multiple interactions of cimetidine and probenecid with valaciclovir and its metabolite acyclovir. Antimicrob Agents Chemother. 2002;46:458-63.

13. Ye J, Liu Q, Wang C, Meng Q, Sun H, Peng J, et al. Benzylpenicillin inhibits the renal excretion of acyclovir by OAT1 and OAT3. Pharmacol Rep. 2013;65:505-12.

14. Ye J, Liu Q, Wang C, Meng Q, Peng J, Sun H, et al. Inhibitory effect of JBP485 on renal excretion of acyclovir by the inhibition of OAT1 and OAT3. Eur J Pharm Sci. 2012;47:341-6.

15. Karla PK, Quinn TL, Herndon BL, Thomas P, Pal D, Mitra A. Expression of multidrug resistance associated protein 5 (MRP5) on cornea and its role in drug efflux. J Ocul Pharmacol Ther. 2009;25:121-32.

16. Hagos $Y$, Wolff NA. Assessment of the role of renal organic anion transporters in drug-induced nephrotoxicity. Toxins. 2010;2:2055-82.

17. Liao TL, Chen YM, Liu HJ, Chen DY. Risk and severity of herpes zoster in patients with rheumatoid arthritis receiving different immunosuppressive medications: a case-control study in Asia. BMJ Open. 2017;7:e014032.

18. Kudaeva FM, Speechley MR, Pope JE. A systematic review of viral exposures as a risk for rheumatoid arthritis. Semin Arthritis Rheum. 2019;48:587-96.

19. Smitten AL, Choi HK, Hochberg MC, Suissa S, Simon TA, Testa MA, et al. The risk of herpes zoster in patients with rheumatoid arthritis in the United States and the United Kingdom. Arthritis Rheum. 2007;57:1431-8.

20. Lee EJ, Jang HN, Cho HS, Bae E, Lee TW, Chang SH, et al. The incidence, risk factors, and clinical outcomes of acute kidney injury (staged using the RIFLE classification) associated with intravenous acyclovir administration. Ren Fail. 2018;40:687-92.

21. Rao S, Abzug MJ, Carosone-Link P, Peterson T, Child J, Siparksy G, et al. Intravenous acyclovir and renal dysfunction in children: a matched case control study. J Pediatr. 2015;166:1462-8. e1-4

22. Becker BN, Fall P, Hall C, Milam D, Leonard J, Glick A, et al. Rapidly progressive acute renal failure due to acyclovir: case report and review of the literature. Am J Kidney Dis. 1993;22:611-5.

23. Vomiero G, Carpenter B, Robb I, Filler G. Combination of ceftriaxone and acycloviran underestimated nephrotoxic potential? Pedia Nephrol. 2002;17:633-7.

24. Gunness P, Aleksa K, Kosuge K, Ito S, Koren G. Comparison of the novel HK-2 human renal proximal tubular cell line with the standard LLC-PK1 cell line in studying drug-induced nephrotoxicity. Can J Physiol Pharmacol. 2010;88:448-55.

25. Ahmad T, Simmonds M, Mclver AG, McGraw ME. Reversible renal failure in renal transplant patients receiving oral acyclovir prophylaxis. Pedia Nephrol. 1994;8: 489-91.

26. Lu H, Han YJ, Xu JD, Xing WM, Chen J. Proteomic characterization of acyclovirinduced nephrotoxicity in a mouse model. PLoS One. 2014;9:e103185.

27. Ma LL, Wu ZT, Wang L, Zhang XF, Wang J, Chen C, et al. Inhibition of hepatic cytochrome $\mathrm{P} 450$ enzymes and sodium/bile acid cotransporter exacerbates leflunomide-induced hepatotoxicity. Acta Pharmacol Sin. 2016;37:415-24.

28. Ceckova M, Reznicek J, Deutsch B, Fromm MF, Staud F. Efavirenz reduces renal excretion of lamivudine in rats by inhibiting organic cation transporters (OCT, Oct) and multidrug and toxin extrusion proteins (MATE, Mate). PLoS One. 2018;13:e0202706.

29. Reznicek J, Ceckova M, Ptackova Z, Martinec O, Tupova L, Cerveny L, et al. MDR1 and BCRP transporter-mediated drug-drug interaction between rilpivirine and abacavir and effect on intestinal absorption. Antimicrob Agents Chemother. 2017:61:e00837-17.

30. Fang Y, Cao W, Liang F, Xia M, Pan S, Xu X. Structure affinity relationship and docking studies of flavonoids as substrates of multidrug-resistant associated protein 2 (MRP2) in MDCK/MRP2 cells. Food Chem. 2019;291:101-9.

31. Ma L, Qin $Y$, Shen $Z$, Bi H, Hu H, Huang $M$, et al. Aristolochic acid I is a substrate of BCRP but not P-glycoprotein or MRP2. J Ethnopharmacol. 2015;172:430-5.

32. Nagle MA, Truong DM, Dnyanmote AV, Ahn SY, Eraly SA, Wu W, et al. Analysis of three-dimensional systems for developing and mature kidneys clarifies the role of OAT1 and OAT3 in antiviral handling. J Biol Chem. 2011;286:243-51.

33. Ivanyuk A, Livio F, Biollaz J, Buclin T. Renal drug transporters and drug interactions. Clin Pharmacokinet. 2017;56:825-92.

34. Nigam SK, Bush KT, Martovetsky G, Ahn SY, Liu HC, Richard E, et al. The organic anion transporter (OAT) family: a systems biology perspective. Physiol Rev. 2015;95:83-123. 
35. El-Sheikh AA, Masereeuw R, Russel FG. Mechanisms of renal anionic drug transport. Eur J Pharmacol. 2008;585:245-55.

36. Xue X, Gong LK, Maeda K, Luan Y, Qi XM, Sugiyama Y, et al. Critical role of organic anion transporters 1 and 3 in kidney accumulation and toxicity of aristolochic acid I. Mol Pharmacol. 2011;8:2183-92.

37. Wolf DL, Rodríguez CA, Mucci M, Ingrosso A, Duncan BA, Nickens DJ. Pharmacokinetics and renal effects of cidofovir with a reduced dose of probenecid in HIV-infected patients with cytomegalovirus retinitis. J Clin Pharmacol. 2003;43: 43-51.

38. Imaoka T, Kusuhara H, Adachi M, Schuetz JD, Takeuchi K, Sugiyama Y. Functional involvement of multidrug resistance-associated protein 4 (MRP4/ABCC4) in the renal elimination of the antiviral drugs adefovir and tenofovir. Mol Pharmacol. 2007;71:619-27.

39. Hiroyuki Kusuhara YS. In vitro-in vivo extrapolation of transporter-mediated clearance in the liver and kidney. Drug Metab Pharmacokinet. 2009;24:37-52.

40. Maeda K, Sugiyama Y. Impact of genetic polymorphisms of transporters on the pharmacokinetic, pharmacodynamic and toxicological properties of anionic drugs. Drug Metab Pharmacokinet. 2008;23:223-35.

41. Watanabe T, Kusuhara H, Maeda K, Shitara Y, Sugiyama Y. Physiologically based pharmacokinetic modeling to predict transporter-mediated clearance and distribution of pravastatin in humans. J Pharmacol Exp Ther. 2009;328:652-62.

42. Kis E, Nagy T, Jani M, Molnar E, Janossy J, Ujhellyi O, et al. Leflunomide and its metabolite A771726 are high affinity substrates of BCRP: implications for drug resistance. Ann Rheum Dis. 2009;68:1201-7.
43. Hulot JS, Villard E, Maguy A, Morel V, Mir L, Tostivint I, et al. A mutation in the drug transporter gene $A B C C 2$ associated with impaired methotrexate elimination. Pharmacogenet Genomics. 2005;15:277-85.

44. Chiasson JM, Fominaya CE, Gebregziabher M, Taber DJ. Long-term assessment of NSAID prescriptions and potential nephrotoxicity risk in adult kidney transplant recipients. Transplantation. 2019; https://doi.org/10.1097/TP.0000000000002689.

45. Pai $A B$, Divine $H$, Marciniak $M$, Morreale A, Saseen JJ, Say K, et al. Need for a judicious use of nonsteroidal anti-inflammatory drugs to avoid communityacquired acute kidney injury. Ann Pharmacother. 2019;53:95-100.

46. Kawase A, Yamamoto T, Egashira S, Iwaki M. Stereoselective inhibition of methotrexate excretion by glucuronides of nonsteroidal anti-inflammatory drugs via multidrug resistance proteins 2 and 4. J Pharmacol Exp Ther. 2016;356:366-74.

47. Nozaki Y, Kusuhara H, Kondo T, Iwaki M, Shiroyanagi $Y$, Nakayama $H$, et al. Species difference in the inhibitory effect of nonsteroidal anti-inflammatory drugs on the uptake of methotrexate by human kidney slices. J Pharmacol Exp Ther 2007;322:1162-70.

48. Kremer JM, Hamilton RA. The effects of nonsteroidal antiinflammatory drugs on methotrexate (MTX) pharmacokinetics: impairment of renal clearance of MTX at weekly maintenance doses but not at 7.5 mg. J Rheumatol. 1995;22:2072-7.

49. Moss DM, Neary M, Owen A. The role of drug transporters in the kidney: lessons from tenofovir. Front Pharmacol. 2014;5:248.

50. Yue Z, Shi J, Li H, Li H. Association between concomitant use of acyclovir or valacyclovir with NSAIDs and an increased risk of acute kidney injury: data mining of FDA adverse event reporting system. Biol Pharm Bull. 2018;41:158-62. 